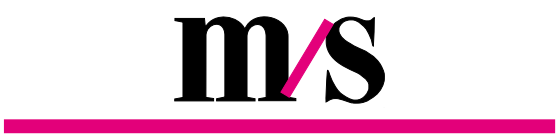

médecinel sciences $2001 ; 17: 539-42$

\title{
LA MISE EN PLACE DES AXES EMBRYONNAIRES DES VERTÉBRÉS : UN PROBLĖME FASCINANT EN PASSE D'ÉTRE RÉSOLU ?
}

a mise en place de l'organisation corporelle des vertébrés s'opère au cours de la gastrulation, un processus impliquant des mouvements cellulaires complexes, au cours duquel les trois feuillets embryonnaires (ectoderme, mésoderme et endoderme) sont formés et ordonnés et les axes embryonnaires (dorso-ventral - DV - et antéro-postérieur - AP) deviennent apparents. De ces deux axes dérive également une identité droite-gauche (DG), à partir de laquelle s'établit l'asymétrie de nombreux organes. Cependant, la mise en place des axes embryonnaires commence bien avant la gastrulation, au cours de l'ovogenèse. L'existence d'une symétrie radiale et d'un axe dit animal-végétatif dans l'œuf non fécondé a été notée depuis longtemps chez les embryons possédant un vitellus. Le premier axe de l'embryon, l'axe DV, dérive de cet axe animal-végétatif, puisqu'il est établi par un mouvement dirigé de molécules déjà présentes dans le cytoplasme de l'ovocyte, donc d'origine maternelle. Ce mouvement est provoqué soit par l'entrée du spermatozoïde (comme chez les amphibiens), soit par gravité (comme chez les oiseaux) [1]. Récemment, il a été établi que des mécanismes analogues de mise en place des axes intervenaient également chez les mammifères $[2,3]$. Les processus moléculaires permettant le passage de cette symétrie radiale à une symétrie bilatérale ont été récemment en partie élucidés. Ces découvertes permettent de bâtir un modèle général de la mise en place des axes chez les vertébrés.

\section{Axe dorso-ventral}

Comment se produit le mouvement dirigé de molécules maternelles ? Cela a été étudié en détail chez le xénope, une grenouille qui a été beaucoup utilisée pour analyser le développement précoce [4]. La pénétration du spermatozoïde dans l'œuf a lieu dans l'hémisphère animal et induit un phénomène de rotation de matériel cytoplasmique cortical dans la direction opposée au point de pénétration et dans le plan défini par ce point et l'axe animalvégétatif. Cette rotation corticale a lieu durant le premier cycle cellulaire et implique la mise en place de réseaux parallèles de microtubules qui vont permettre un déplacement, rapide (de l'ordre de $40 \mu \mathrm{m} / \mathrm{min}$ ) et d'environ $90^{\circ}$ vers l'équateur, de petites vésicules initialement localisées au pôle végétatif. Ces vésicules transportent vraisemblablement des molécules qui vont participer à la définition du côté dorsal de l'embryon [4].

Quelles sont ces molécules ? Lorsque I'œuf fécondé est irradié par une lumière UV, la migration corticale ne
S. Schneider-Maunoury, P. Charnay : Inserm
$U$. 368, École normale supérieure, 46, rue d'Ulm, 75230 Paris Cedex 05, France. 
peut avoir lieu car les réseaux de microtubules sont rendus non fonctionnels et l'embryon ne développe aucune caractéristique dorsale : il est ventralisé. II est alors possible, par injection d'ARN messagers codant pour différentes protéines, d'identifier celles qui permettent de restaurer l'identité dorsale. Cette méthode a permis d'impliquer les composants de la voie de signalisation Wnt dans la mise en place du territoire dorsal $\left(\mathrm{m} / \mathrm{s} 1997, \mathrm{n}^{\circ} 2\right.$, p. 222) $[4,5]$. Les protéines Wnt constituent une famille de protéines secrétées qui se fixent à des récepteurs spécifiques enchassés dans la membrane cellulaire et activent une voie de transduction intracellulaire. Un acteur essentiel de cette voie est la $\beta$-caténine. Sous I'action du signal Wnt, cette protéine du cytosquelette est stabilisée et transportée dans le noyau des cellules où elle s'associe à des facteurs de transcription de la famille TCF pour activer l'expression de gènes spécifiques. Au cours de la rotation corticale, les molécules transportées permettent l'activation de la $\beta$-caténine, qui est essentielle à la définition du côté dorsal de l'embryon. Cependant, l'identité des molécules activatrices transportées n'est pas encore établie. La protéine Wnt impliquée n'a pas été identifiée, mais des expériences très récentes montrent qu'un récepteur de Wnt, Frizzled-7, est nécessaire à la formation de l'axe DV.

Quelles sont les conséquences de la relocalisation des éléments de la voie Wnt ? Chez les embryons ventralisés par le traitement UV, les vésicules corticales immobilisées au pôle végétatif conduisent à la stabilisation et à la translocation locale de la $\beta$-caténine, mais sans développement d'un côté dorsal. Celui-ci requiert que I'activation des gènes commandés par la $\beta$-caténine ait lieu à proximité de la zone équatoriale, là où le mésoderme est engendré, à la jonction de l'ectoderme et de l'endoderme. En effet, I'autre composant de la mise en place de l'axe dorso-ventral est l'induction du mésoderme par l'endoderme qui se produit dans la région équatoriale, et implique une autre famille de molécules secrétées,
Au stade blastula, les gènes $X n r$ sont exprimés en un gradient DV décroissant dans l'endoderme, sous contrôle positif conjoint de la $\beta$-caténine, localisée du côté dorsal, et d'inducteurs du mésoderme, présents au pôle végétatif. Dans la région dorso-végétative, appelée centre de Nieuwkoop (m/s 1994, $n^{\circ} 8-9$, p. 854), les $X n r$ sont secrétés en grande quantité et sont capables d'induire du mésoderme dorsal dans la région équatoriale adjacente. En revanche, hors de la zone dorsale, les cellules équatoriales sont exposées à de faibles doses de $\mathrm{X} n \mathrm{r}$ et en conséquence donnent naissance à du mésoderme ventral. Le rôle de l'accumulation de $\beta$-caténine dans I'établissement de l'identité dorsale ne se limite pas à l'induction d'un haut niveau de $X n r$ : elle va également participer à une dorsalisation directe des trois feuillets embryonnaires [4].

Le mésoderme dorsal formé par l'action conjointe de la $\beta$-caténine et des $X n r$ acquiert des propriétés remarquables, qui lui ont valu le nom d'organisateur de Spemann ( $\mathrm{m} / \mathrm{s} 1992, n^{\circ} 5$, p. 483). Cette région, transplantée dans la partie ventrale d'un autre embryon, est capable d'induire la formation d'un deuxième embryon complet. Les cellules qui constituent l'organisateur ne participent que partiellement à la formation du nouvel embryon, mais sont capables d'induire la région ventrale de l'embryon hôte à former du tissu dorsal, et ainsi à mettre en place de nouveaux axes DV et AP. Comment I'organisateur fonctionne-t-il pour induire la dorsalisation des tissus hôtes ? En fait, l'organisateur secrète de multiples facteurs, antagonistes de différents types de protéines signalisatrices. Parmi ces facteurs, on trouve des antagonistes des BMP (bone morphogenic protein) (m/s 1996, $n^{\circ} 3$, p. 364), une famille de molécules sécrétées essentielles pour l'identité ventrale. Ces antagonistes, la chordine, la noggine et la follistatine, vont également participer à l'induction neurale, qui correspond à une dorsalisation de l'ectoderme. Une deuxième catégorie de protéines dorsalisantes secrétées par l'organisateur, les membres de la famille sFRP, comme Frzb-1, et les membres de la famille Dickkopf (Dkk) (m/s 1998, $\left.n^{\circ} 10, p .1144\right)$ agissent comme inhibiteurs des protéines Wnt [4]. En effet, certains Wnt, comme X wnt-8, ont une activité ventralisante dans le mésoderme. Ce dernier exemple illustre ainsi la complexité d'un processus lors duquel les mêmes types de molécules, ici les Wnt, sont réutilisés plusieurs fois dans des rôles différents et apparemment contradictoires, à différents stades de développement ou dans différents tissus.

\section{Axe antéro-postérieur}

$L$ 'axe AP se forme plus tard que l'axe DV et dépend de ce dernier. Chez les amphibiens, la greffe de l'organisateur dans la région ventrale d'un embryon hôte est non seulement susceptible d'induire la formation de structures dorsales, mais également de les organiser dans l'espace pour former un nouvel embryon avec un axe AP complet. L'organisateur fournit donc tous les signaux nécessaires à la formation de cet axe.

Les mécanismes de l'établissement des coordonnées AP de l'embryon ont été bien étudiés en ce qui concerne le neurectoderme, le futur système nerveux central. Plusieurs théories ont été émises. L'un de ces modèles, établi par Nieuwkoop en 1952 et appelé modèle d' « activationtransformation », était encore récemment admis. II proposait que I'organisateur envoie deux types de signaux successifs à l'ectoderme, un signal induisant la formation de tissu neural de type antérieur, et un signal permettant la postériorisation progressive de ce tissu. Le modèle d'activation-transformation a été conforté par la découverte de l'activité des inhibiteurs de BMP - chordine, noggine et follistatine - présents dans l'organisateur. En effet, ces molécules induisent toutes la formation de tissu neural de caractère antérieur. En revanche, les molécules capables de donner à ce tissu neural un caractère postérieur, n'ont pas généralement d'activité neuralisante (c'est le cas des rétinoïdes et de certaines protéines Wnt), à l'exception des FGF (fibroblast growth factor), pour lesquels la question reste posée [7]. Plus récemment, la validité de ce 
modèle a été remise en question par la découverte chez l'embryon de souris d'une autre région nécessaire à la formation du cerveau antérieur [8]. Cette région est localisée au niveau de la partie antérieure de l'endoderme viscéral ( $A V E)$, un tissu qui ne participe pas à la formation de l'embryon lui-même. Elle peut être mise en évidence par l'expression spécifique de plusieurs gènes régulateurs, avant même le début de la gastrulation. Quand cette région est éliminée par microchirurgie, les embryons qui en résultent n'ont pas de tête. De plus, l'inactivation de plusieurs gènes exprimés dans I'AVE, tels que 0 tx 2 et $\operatorname{Lim} 1$, conduit également à des embryons sans tête. Des études très récentes suggèrent que l'AVE fonctionne en empêchant I'acquisition d'une identité postérieure [9].

Un équivalent fonctionnel de I'AVE a été mis en évidence chez les amphibiens [7]. II correspond à une population cellulaire située dans une zone profonde de l'organisateur, destinée à donner du mésendoderme antérieur. Plusieurs molécules secrétées ont été impliquées dans l'induction de la tête. Parmi elles se trouvent des molécules secrétées antagonistes des Wnt et des BMP, de type Dkk ou Cerberus, ainsi que des molécules signalisatrices de type Nodal/ Xnr et FGF. U ne autre région à activité inductrice a été mise en évidence chez le poisson. Ce territoire, situé ventralement à l'organisateur, permet d'induire la formation de tissu neural à caractère AP intermédiaire : le rhombencéphale ou cerveau postérieur. Ainsi, ces études récentes montrent que, contrairement au modèle initial de Nieuwkoop, différents signaux, provenant du mésoderme ou de l'endoderme, sont nécessaires pour induire les différents niveaux antéro-postérieurs du tube neural. Ces différentes activités sont regroupées chez les amphibiens dans l'organisateur de Spemann, lui conférant ainsi les propriétés remarquables mises en évidence par l'embryologie classique.

Comment s'établissent les coordonnées AP dans l'organisateur ? On n'a pas pour le moment de réponse claire à cette question. Une avancée récente dans ce domaine est la découverte du rôle de la voie de signalisation Nodal/ Xnr dans la régionalisation AP du mésoderme axial, dérivé de l'organisateur. Un haut niveau de Nodal est requis pour former du mésoderme axial antérieur (précordal) alors qu'un niveau plus faible donne naissance à du mésoderme axial postérieur (cordal) (m/s 1999, n², p. 1464) [10].

\section{L'asymétrie droite-gauche}

Vu de l'extérieur, le corps humain (et celui des vertébrés en général) présente une symétrie DG presque parfaite (m/s 1999, $n^{\circ} 3$, p. 417). Cependant, cette symétrie apparente cache des asymétries internes de nombreux organes, et diverses anomalies de I'orientation DG sont connues chez I'homme. Contrairement aux asymétries AP et DV qui deviennent apparentes pendant la gastrulation, l'asymétrie DG ne devient visible que plus tard, avec la courbure asymétrique du tube cardiaque et du tube digestif. Cependant, la caractérisation de gènes exprimés de manière asymétrique au cours de la gastrulation a montré que l'asymétrie est mise en place au moins dès ce stade, et que certains mécanismes sont conservés chez tous les vertébrés [11]. Par exemple, la voie de signalisation Nodal/ Xnr est impliquée chez tous les vertébrés, ainsi que des gènes cibles de cette voie tels le gène à homéoboîte Pitx2 et les gènes lefty, codant pour des antagonistes de Nodal. Cette voie est d'ailleurs également impliquée dans l'établissement plus tardif de l'asymétrie droitegauche dans le cerveau [12]. En revanche, un consensus n'a pas été trouvé en ce qui concerne l'établissement de l'asymétrie [11]. Chez la souris, le gène nodal, tout d'abord exprimé dans tout le nœud (I'équivalent de l'organisateur de Spemann chez cette espèce), voit son expression progressivement restreinte au côté gauche. Cette restriction est supposée résulter d'un flux de fluide vers la partie gauche du nœud, dû au mouvement de rotation de cils présents sur la face ventrale des cellules du nœud, les cils nodaux. En effet, plusieurs mutations qui affectent l'asymétrie droite-gauche chez la souris touchent des protéines appelées moteurs moléculaires, permettant le transport intracellulaire d'autres protéines le long des microtubules. On peut citer par exemple la dynéïne LRD, ou deux protéines de la famille des kinésines, KIF3A et KIF3B. Ces mutations affectent également la formation ou la motilité des cils nodaux. II a donc été proposé que l'asymétrie de l'expression du gène nodal soit créée par un flux de la protéine nodal ellemême vers le côté gauche, dû au mouvement directionnel des cils nodaux. Ce modèle est attrayant car il permet d'expliquer comment I'asymétrie se met en place. Cependant, cette hypothèse n'est pas en accord avec de nombreuses observations faites chez d'autres vertébrés, indiquant que l'asymétrie est créée en dehors de l'organisateur. II est donc possible que les mécanismes gouvernant l'établissement de l'asymétrie droite-gauche ne soient pas conservés entre les différentes classes de vertébrés. Alternativement, il se pourrait que les cils nodaux chez la souris ne servent qu'à maintenir une asymétrie préexistante.

\section{Conclusions}

Les avancées récentes dans la compréhension de la mise en place des axes embryonnaires découlent de la définition précise, à la fois spatiale et temporelle, des territoires ayant une activité inductrice, mais aussi et surtout de l'identification des voies de signalisation impliquées dans ces inductions. En ce qui concerne ce dernier point, deux caractéristiques importantes méritent d'être soulignées. D'une part, ces voies de signalisation sont en nombre restreint et sont donc réutilisées lors d'étapes successives. D'autre part, l'implication de nombreux antagonistes de ces voies montre que le contrôle précis de l'inhibition de signaux inducteurs constitue un aspect essentiel de la régulation du développement précoce

\section{Remerciements}

Les auteurstiennent à remercier Frédéric Rosa, Christine Vesque et Marion Wassef pour la lecture critique de cet article. 


\section{RÉFÉRENCES}

1. Eyal-Giladi H. Establishment of the axis in chordates: facts and speculations. Development $1997 ; 124$ : 2285-96.

2. Piotrowska K, Zernicka-Goetz M. Role for sperm in spatial patterning of the early mouse embryo. Nature $2001 ; 409$ : 517-21.

3. Gardner RL. Polarity in early mammalian development. Curr Opin Genet Dev 1999 ; 9 : 417-21.

4. De Robertis EM, Larrain J, O elgeschlager $M$, Wessely 0 . The establishment of Spemann's organizer and patterning of the vertebrate embryo. Nat Rev Genet $2000 ; 1$ : 171-81.
5. Sokol SY. Wnt signaling and dorso-ventral axis specification in vertebrates. Curr 0 pin Genet Dev $1999 ; 9$ : 405-10.

6. Schier AF, Shen MM. Nodal signalling in vertebrate development. N ature $2000 ; 403$ : 385-9.

7. Gamse I, Sive H. Vertebrate anteroposterior patterning: the $X$ enopusneuroectoderm as a paradigm. Bioessays $2000 ; 22$ : 976-86.

8. Beddington RS, Robertson EJ. Anterior patterning in mouse. T rends Gen et 1998; 14 : 277-84

9. Perea-Gomez A, Rhinn M, Ang SL. Role of the anterior visceral endoderm in restricting posterior signals in the mouse embryo. Int ] Dev Biol 2001 ; 45 : 311-20.
10. Gritsman K, Talbot WS, Schier AF. Nodal signaling patterns the organizer. Development $2000 ; 127: 921-32$.

11. Supp DM, Potter SS, Brueckner M. Molecular motors: the driving force behind mammalian left-right development. T rends Cell Biol $2000 ; 10: 41-5$.

12. Concha ML, Burdine RD, Russell C, Schier AF, Wilson SW. A Nodal signaling pathway regulates the laterality of neuroanatomical asymmetries in the zebrafish forebrain. Neuron $2000 ; 28$ : 399-409.

\section{TIRÉS À PART}

S. Schneider-Maunoury et P. Charnay. 